\title{
Overestimation of vertical movement
}

\author{
A. A. LANDAUER, C. A. RHINE AND L. RUMIZ \\ THE UNIVERSITY OF WESTERN AUSTRALIA
}

Forty-eight Ss tracked the movement of a dot of light which was presented in the horizontal/vertical plane under open viewing conditions but without being able to monitor visually their drawing. The vertical movement components were significantly overestimated, both when Ss tracked in the upright or level plane. These findings are believed to be a manifestation of the vertical-horizontal illusion.

In the course of a prior investigation of autokinetic movement it became apparent that Ss responded by drawing more movement in the vertical plane than in the horizontal one. Similar findings have been reported by Stern (1964), who attempted to explain the vertical movement preference in terms of Künnapas' (1959) theory.

If the preference for vertical movement in autokinesis is due to an effect of the vertical-horizontal lllusion, it can be postulated that in tracking the real movement of a dot of light the vertical movement components would also be overestimated. This effect should be particularly enhanced if Ss are unable to monitor visually their tracking task.

\section{Subjects}

Forty-eight male undergraduates with uncorrected vision and with a mean age of 20.5 years served as volunteer Ss. None of the Ss was familiar with the phenomenon being investigated. Three Ss were lefthanded and used their left hand in performing the tracking task.

\section{Apparatus and Procedure}

Two $8 \mathrm{~mm}$ moving films $\left(F_{1}\right.$ and $\left.F_{2}\right)$, presented at 16 frames per sec and each of 42 sec duration were made. When viewed in the experimental condition, each film showed a white dot $(0.38 \mathrm{in}$. in diameter) moving on a dark gray background along the tracks shown in Fig. 1. In the actual films the movement was somewhat irregular and the corners were rounded, but the ratio of vertical to horizontal movement components was 0.97 for $F_{1}$ and 1.00 for $F_{2}$. In both films the movement of the dot started from the center of an $11.6 \mathrm{in.} \mathrm{high} \mathrm{and} 15.8 \mathrm{in}$. wide screen, and the tracks were $14.5 \mathrm{in}$. $\left(F_{1}\right)$ and $15.5 \mathrm{in}$. $\left(F_{2}\right)$ long. The films were presented by an automatic moving film projector (Technicolor Movie Vision projector, Model $600)$.

Two tracking positions were used, one in which the board on which movement had to be drawn was in the upright plane (U.P.), and one in which the drawing board was in the level plane (L.P.). The position of the board could be horizontally and vertically adjusted to allow for a comfortable position during the tracking task. A stack of 14.75 in. high $\times 23$ in. long paper was pinned to the board. These sheets had a
$1 / 4$ in. central hole which represented the starting position for the tracking task. A felt pen with a multidirectional point, filled with quick drying ink, permitted effortless movement without pressure, and produced fine and clear tracings. A suitably placed board obscured S's view of the paper on which he had to draw the movement of the dot.

The experimental room was illuminated by natural light and by a $200 \mathrm{~W}$ incandescent lamp. Subjects were given formalized Instructions which stressed that the experiment measured accuracy of tracking a moving stimulus. They were told that when the dot appeared at the center of the screen, they were to begin to draw its movement as accurately as they could, keeping their attention on the screen at all times. They were also warned not to lift the pen off the paper during the tracking task.

The 48 Ss were tested Individually and were randomly allocated into four groups of equal size, two for the U.P. tracking task and two for the L.P. presentation. The first group in each of the tracking positions were shown $F_{1}$ followed by $F_{2}$, while in the other group the order of film presentation was reversed.

Subjects were seated in front of and at eye level with the screen. Viewing distance was $14 \mathrm{ft}$, observation was binocular, and head movement was restricted by an adjustable chin rest.

After S had tracked the movement of the first film, the paper on which the movement was traced was removed and the second film was shown. In all cases the first film served as practice trial and was not scored.

The following scoring procedure was adopted. The drawing was placed on a sheet of $1 / 4$ in. graph paper. The number of horizontal and vertical squares through
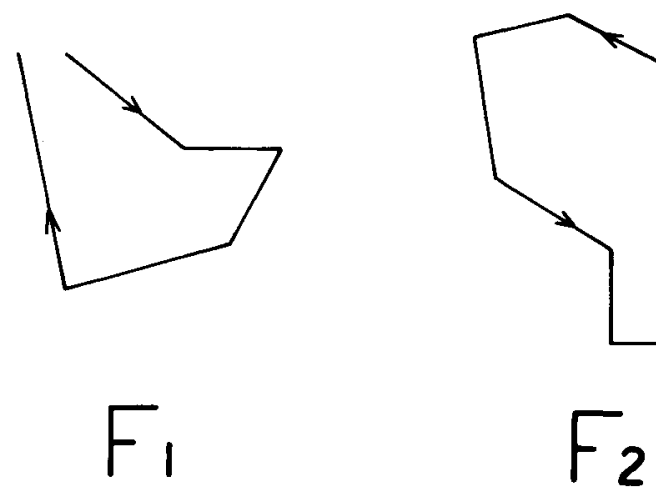

Fig. 1. Track of dot on films 1 and 2. 
Table 1

\begin{tabular}{c|ccc} 
& U.P. & L.P. & Both Planes \\
\hline$F_{1}$ & 113.1 & 124.0 & 118.55 \\
$F_{2}$ & 127.2 & 130.5 & 128.85 \\
Both Films & 120.15 & 127.25 & 123.70 \\
\hline
\end{tabular}

Mean ratios obtained.

which the tracking passed were counted, and a ratio for each tracking was calculated by dividing the vertical movement components (number of vertical squares) by the horizontal movement components (number of horizontal squares), and multiplying the quotient by $100(\mathrm{~V} / \mathrm{H} \times 100)$. This ratio was used in the evaluation of the results.

\section{Results and Discussion}

Table 1 shows the mean results obtained. An analysis of variance of the data showed that there was no significant difference between the ratios obtained with the two films, between the ratios obtained with the two presentations of the drawing board (U.P. and L.P.), nor was there any significant interaction. For this reason the results of the four experimental groups were combined for the testing of the main hypothesis.

As there were equal amounts of vertical and horizontal movement components in both films, a veridical tracking of the movement of the dot should have re- sulted in a ratio which did not significantly depart from 100. A $t$ test was made to ascertain whether the grand mean of $\mathbf{1 2 3 . 7}$ differed significantly from the hypothesized mean of 100 . The value for $t$ for this difference was 6.26 (df $=47, p<.001) .^{1}$

These results clearly demonstrate that in the experimental conditions used here there is considerable vertical overestimation in tracking movement. This overestimation is approximately equal to the amount of the vertical-horizontal illusion commonly reported (Künnapas, 1959) and the results may be a manifestation of this illusion. It may well be the case that even if $S$ could monitor visually the moving dot and the board on which the tracking is made, an overestimation of vertical movement will occur.

\section{References}

KUNNAPAS, T. M. Visual field as a frame of reference. Stockholm: Almquist \& Wiksells, 1959.

STERN, R. M. Long-term observation of the autokinetic illusion: frequency and direction of movement. Percept. mot. Skills, 1964, $18,825-830$.

\section{Note}

1. After obtaining this $t$ value the departure of each separate mean from the hypothesized mean was checked. It was found that each differed significantly from the hypothesized mean at $p<0.05$ or better. These findings are also in agreement with the incidental (nonpostulated) finding of another experiment in which, in addition to the two films used in this investigation, other films having two horizontal or vertical dots and moving along different tracks, were used. 